\title{
Effectiveness of Mini-Implant for the Reduction of Mandibular Fracture
}

\author{
Nam-Ho Kim, Jeong-Uk Heo, Jun-Sub Park \\ Goodwill Dental Hospital, Busan, Korea
}

\begin{abstract}
Purpose: This study sought to verify the usefulness of mini-implant and surgical steel wire in the treatment of mandibular fracture through the objective identification of the change of bone structure and bone density before and after reduction by evaluating radiological change through fractal analysis when mandibular fracture is treated using mini-implant and surgical wire.

Materials and Methods: This study looked at 45 patients (males: 38, female: 7) diagnosed with mandibular fracture in the oral and maxillofacial surgery division of Chung-Ang University Dental Hospital and who received open reduction and intra-osseous fixation.

Result: The average fracture dimension values were higher for the group of the patients who had mini-implants and surgical wire treatment.

Conclusion: Based on the results of the study on the usefulness of the reduction technique using mini-implant and surgical steel wire in the treatment of mandibular fracture through the fractal analysis method, the reduction technique using mini-implant and surgical steel wire is regarded as an effective method of minimizing the gap between mandibular fracture fragments.
\end{abstract}

Key Words: Fractal; Mandibular fracture; Mini-implant

\section{Introduction}

The treatment of mandibular fracture aims at the anatomical location recovery of fracture fragment, perfect occlusion, and restoration of mandibular function ${ }^{1}$. As the treatment method for these, the non-invasive method wherein only intermaxillary fixation is performed and invasive method wherein skeletal anchorage is performed using a metal plate have long been used. As for open reduction, the approach and reduction method differ according to the fracture site, condition, displacement degree, and surgical device and skill of the operator. This is because various cases occur according to the

Corresponding Author: Jeong-Uk Heo

Goodwill Dental Hospital, 28 Geumgok-daero, Buk-gu, Busan 616-817, Korea

TEL : +82-51-330-6000, FAX : +82-51-330-6098, E-mail : eqdent@hanmail.net

Received for publication November 28, 2012; Returned after revision April 23, 2013; Accepted for publication April 30, 2013

Copyright $\odot 2013$ by Korean Academy of Dental Science

(c) This is an open access article distributed under the terms of the Creative Commons Attribution Non-Commercial License (http://creativecommons.org/licenses/ by-nc/3.0) which permits unrestricted non-commercial use, distribution, and reproduction in any medium, provided the original work is properly cited. 
fracture site and condition, and the best method may vary depending on the case and the skill of the operator ${ }^{2}$. In the case of open reduction, miniplate fixation, wherein metal plate can be fixed with an intraoral approach that does not leave scars on skin is widely used ${ }^{3}$. When fixing the fracture fragment with this metal plate, we need a method of holding and maintaining the fracture fragment to fix the metal plate on the fracture fragment easily without causing any change of fracture fragment $t^{4)}$. This is because the principle of mandibular fracture reduction is that, after the reduction of fracture fragment to the original location, the contact area between fracture fragments should be kept as stable as possible during the histopathological healing stage of fracture fragment consisting of inflammatory phase, reparative phase, and remodeling phase $^{5}$. For this, we need a method of minimizing the gap between fracture fragments and to minimize the movement of fracture fragments with proper fixation. The reduction using mini-implant surgical steel wire is a new method that has reduced the time and difficulty for the reduction of two fracture fragments to the original location during the open reduction of mandibular fracture; it can be used effectively in a short period of time $e^{5}$. The temporary use of mini-implant and surgical steel wire before intraosseous fixation has minimized the gap between fracture fragments ${ }^{6}$.

The evaluation of reduction in the treatment of fracture is made with radiography at first. We need a method for objective evaluation since the evaluation of the contact area between fracture fragments differs according to the shooting device type, exposure time, thickness of osseous tissue and density and thickness of overlapped soft tissue ${ }^{7,8)}$.

Nowadays, fractal analysis is being used to evaluate bone density and form more accurately. This is known to be able to evaluate the change of bone structure or bone density more objectively without any influence of variables based on the basic notion that fractal dimension remains the same, although visual form is influenced if the contrast of image changes ${ }^{9-111}$.

For this reason, this study sought to verify the usefulness of mini-implant and surgical steel wire in the treatment of mandibular fracture through the objective identification of the change of bone structure and bone density before and after reduction by evaluating radiological change through fractal analysis when mandibular fracture is treated using mini-implant and surgical steel wire.

\section{Materials and Methods}

\section{Object of the Study}

This study looked at 45 patients (males: 38, females: 7) diagnosed with mandibular fracture in the oral and maxillofacial surgery division of Chung-Ang University Dental Hospital and who received open reduction and intra-osseous fixation; the average age was 37.8 for males and 29.4 for females. The patients listened to sufficient explanation about this study and gave their consent before being selected as object of the study. Out of a total of 45 patients, 39 had more than two mandibular fractures with anatomically different location. From these, a total of 60 fracture sites included in body fracture, angle fracture, ascending ramus fracture, and subcondylar fracture were selected as the object of study. For 30 sites, reduction using mini-implant and surgical steel wire was applied (group A); for the remaining 30 sites, mini-implant and surgical steel wire were not applied (group B) Table 1.

Table 1. Number of fracture sites

\begin{tabular}{lcc}
\multicolumn{1}{c}{ Site } & Group A $(\mathrm{n}=30)$ & Group B $(\mathrm{n}=30)$ \\
\hline Body & 6 & 6 \\
Angle & 15 & 14 \\
Ascending ramus & 5 & 6 \\
Subcondylar & 4 & 4 \\
\hline
\end{tabular}

Group A: reduction with mini-implant and wire, Group B: conventional reduction. 


\section{Method of Study}

1) Radiography Shooting

We collected panoramic radiographs of the mandibular fracture site during the first examination, 1 day after surgery, and average of 5.8 weeks after surgery. The image of digital panoramic radiographs measures $1976 \times 976$ pixels and the pictures were saved as bitmap image file.

2) Performance of Open Reduction and Intra-Osseous Fixation

(1) Performance of open reduction and intraosseous fixation using mini-implant and surgical steel wire

We made a $5 \mathrm{~mm}$ incision on the facial skin corresponding to the location of the fracture line to be opened to the inside of the mouth and located a driver inside of the mouth toward the center of the fracture line to place the mini-implant.

In addition, we selected an appropriate location adjacent to the fracture line and placed two miniimplants upward of the inferior alveolar nerve and two mini-implants downward of the inferior alveolar nerve. After that, we removed the driver. Here, through manual operation, bone reduction was performed to minimize the gap between proximal segment and distal segment, followed by intermaxillary fixation. If the upward miniimplants and the downward mini-implants of inferior alveolar nerve are ligated with surgical wire, the gap between proximal segment and distal segment almost disappears (Fig. 1). After checking the fracture line and the completed bone reduction, we placed the trans-buccal set again on the cut skin area for the placement of mini-implant and performed intra-osseous fixation.

(2) Performance of conventional open reduction and intra-osseous fixation

In the case of conventional open reduction, we performed reduction using manual operation and bone reduction forceps, followed by intra-osseous fixation with intramaxillary ligation.

3) Image Processing and Fractal Dimension Analysis

With the fracture line on the selected panoramic radiograph as the center, we selected the maximum superior area (S) and inferior area (I) of the fracture line that does not include the cortical bone and adjacent anatomical structures and right middle point $(\mathrm{M})$ between the maximum superior area and inferior area as the region of interest (ROI) at $30 \times 30$ pixels (Fig. 2). With enough time difference, we selected the ROI three times $(0,1,2)$ and obtained each fractal dimension value. Afterward, we set the average of those three values as the fractal dimension value of the corresponding areas. Moreover, as the ROI of $30 \times 30$ pixels, we selected the near area $(\mathrm{N})$ of the right middle point on the fracture line whose trabecular pattern is clear and which is regarded as normal mandible. We applied Gaussian filter (sigma=35 pixel) using Photoshop (version 7.0.1; Adobe Systems Inc., Mountain View, CA, USA) of Adobe to the ROI images selected using the method of White and Rudolph ${ }^{12)}$ and

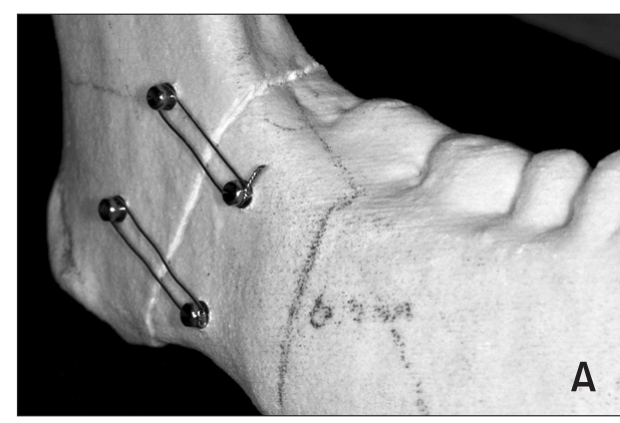

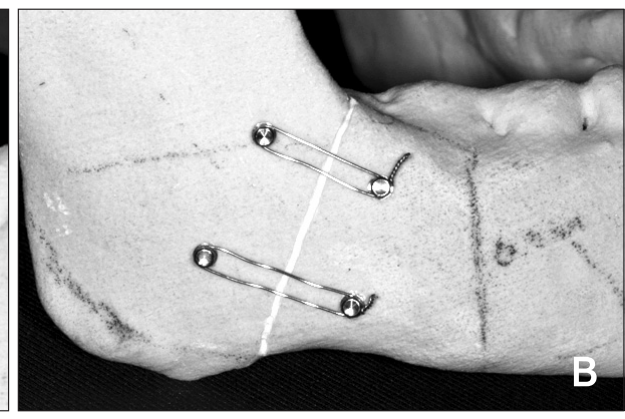

J Korean Dent Sci 2013;6(1):4-12
Fig. 1. Gap-free reduction with 4 mini-implants and surgical wires on simulated model (A) and replica case (B). 
blurred them to get the structures whose difference of radiographical density was huge. We also subtracted this blurred image from original images using the Image J program (1.43u, National Institute of Health, Bethesda, MD, USA), and then added 128 and changed them to binary image. After reducing

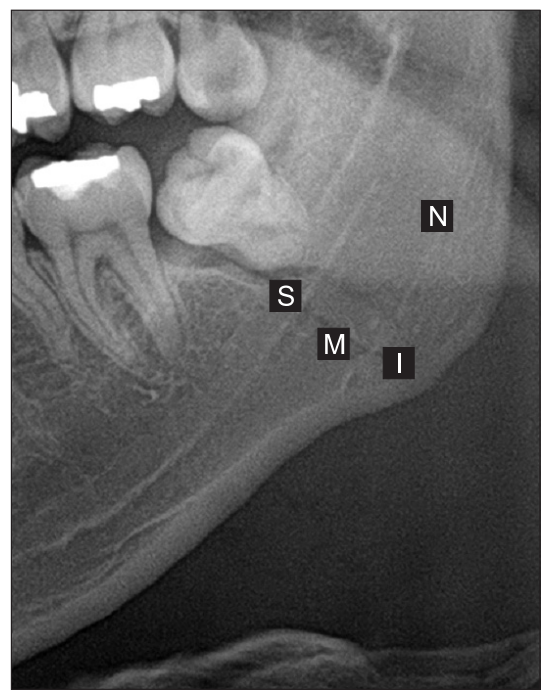

Fig. 2. Region of interest (ROI) on the panoramic radiograph. $\mathrm{N}$ : ROI of normal bone, S: ROI of superior area on the fracture line, $\mathrm{M}$ : ROI of middle area on the fracture line, I: ROI of inferior area on the fracture line. the noise form of this binary image through erosion and dilation, we changed it to a skeletonized image for processing (Fig. 3).

Using the Image J program, with box-counting method, we calculated the fractal dimension value of the images obtained through image processing.

\section{4) Statistical Analysis}

For each fractal dimension value, we used the SPSS Statistics program (version 17.0; SPSS Inc., Chicago, IL, USA). First, as the comparative analysis of each ROI before surgery, 1 day after surgery, and 5.8 weeks after surgery, we looked into whether they had significant difference in fractal dimension by performing repeated measures ANOVA. Likewise, to compare the fractal dimension values of ROI areas on the fracture line according to the method of surgery, we carried out statistical processing with the Mann-Whitney rank sum test and analyzed the significance of the changes in ROI fractal dimension value on the fracture line using sign-rank test.

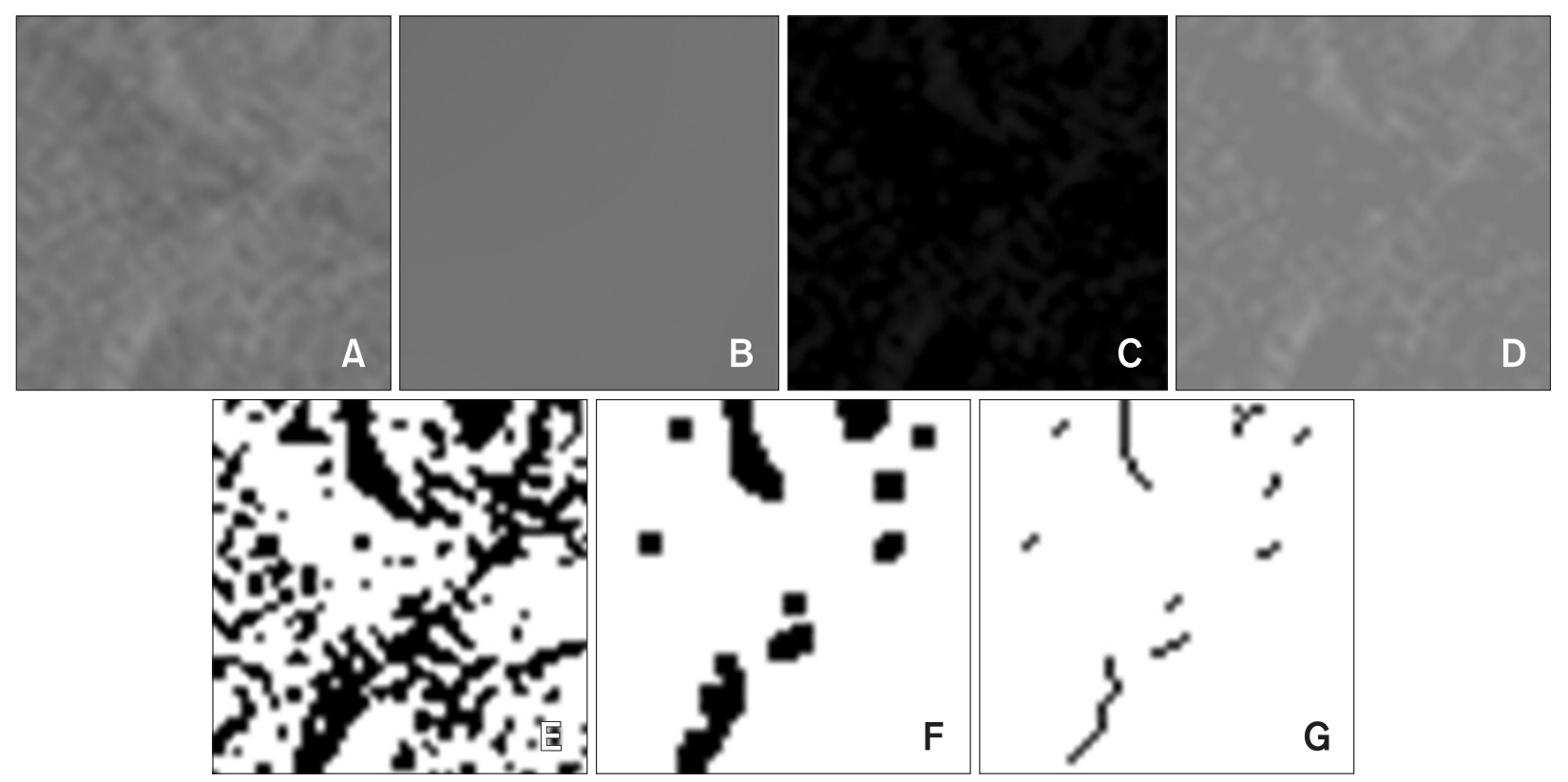

Fig. 3. Image transforming process (A: original image, B: gaussian blur, C: subtract, D: add, E: binary, F: erosion, dilation, G: skeletonize). 


\section{Result}

\section{Changes of Fractal Dimension on the Fracture Line}

The fractal dimension values of ROI on the fracture line in the first examination and 5.8 weeks on the average after performing open reduction and intra-osseous fixation have increased on the average regardless of the surgery method. The signrank test showed a significant result at $\mathrm{P}<0.001$. Therefore, the fractal dimension values in the first examination and 5.8 weeks on the average after surgery were deemed to have statistically significant difference. Moreover, since the median of the difference was positive number, we found that the fractal dimension value on the fracture line had significantly increased regardless of surgery method.

\section{Changes of Fractal Dimension according to} Time Flow

The average fractal dimension values of $\mathrm{CS}_{0}$,
$\mathrm{CM}_{0}, \mathrm{CI}_{0}$, and $\mathrm{CN}_{0}$ in the radiograph in the first examination of the patients to whom mini-implant and surgical wire technique were applied were $0.7163 \pm 0.1407,0.7120 \pm 0.1486,0.6916 \pm 0.1230$, and $1.0922 \pm 0.2237$, respectively. The average fractal dimension values of $\mathrm{CS}_{1}, \mathrm{CM}_{1}, \mathrm{CI}_{1}$, and $\mathrm{CN}_{1}$ in the radiograph taken 1 day after surgery were $0.9675 \pm 0.1034,0.9551 \pm 0.1126,0.9809 \pm 0.0985$, and $1.1122 \pm 0.2112$, respectively. Finally, the average fractal dimension values of $\mathrm{CS}_{2}, \mathrm{CM}_{2}, \mathrm{CI}_{2}$, and $\mathrm{CN}_{2}$ in the radiograph taken 5.8 weeks on the average after surgery were $1.0123 \pm 0.1253,1.0003 \pm 0.1211$, $0.9905 \pm 0.2093$, and $1.1771 \pm 0.2105$, respectively. The average fractal dimension values of $\mathrm{S}_{0}, \mathrm{M}_{0}, \mathrm{I}_{0}$, and $\mathrm{N}_{0}$ in the radiograph in the first examination of patients who underwent conventional open reduction and intra-osseous fixation were $0.6966 \pm 0.1378,0.6701 \pm 0.1501,0.6737 \pm 0.1136$, and $1.1353 \pm 0.1623$, respectively. On the other hand, the average fractal dimension values of $S_{1}, M_{1}, I_{1}$, and $\mathrm{N}_{1}$ in the radiograph taken 1 day after surgery were

Table 2. Fractal dimension selected at first diagnosis

\begin{tabular}{rcccccccc}
\hline & $\mathrm{N}_{0}$ & $\mathrm{~S}_{0}$ & $\mathrm{M}_{0}$ & $\mathrm{I}_{0}$ & \multicolumn{1}{c}{$\mathrm{CN}_{0}$} & \multicolumn{1}{c}{$\mathrm{CS}_{0}$} & $\mathrm{CM}_{0}$ & $\mathrm{Cl}_{0}$ \\
\hline \multirow{2}{*}{ Mean $\pm \mathrm{SD}$} & $1.1353 \pm$ & $0.6966 \pm$ & $0.6701 \pm$ & $0.6737 \pm$ & $1.0922 \pm$ & $0.7163 \pm$ & $0.7120 \pm$ & $0.6916 \pm$ \\
& 0.1623 & 0.1378 & 0.1501 & 0.1136 & 0.2237 & 0.1407 & 0.1486 & 0.1230 \\
\hline
\end{tabular}

$\mathrm{N}_{0}, \mathrm{~S}_{0}, \mathrm{M}_{0}, \mathrm{I}_{0}$ : dimension with conventional technique at first diagnosis, $\mathrm{CN}_{0}, \mathrm{CS}_{0}, \mathrm{CM}_{0}, \mathrm{CI}_{0}$ : dimension with mini-implant and surgical wire at first diagnosis, SD: standard deviation.

Table 3. Fractal dimension selected at 1 day postoperative

\begin{tabular}{rrrrrrrrr}
\hline & $\mathrm{N}_{1}$ & \multicolumn{1}{c}{$\mathrm{S}_{1}$} & $\mathrm{M}_{1}$ & $\mathrm{I}_{1}$ & \multicolumn{1}{c}{$\mathrm{CN}_{1}$} & \multicolumn{1}{c}{$\mathrm{CS}_{1}$} & $\mathrm{CM}_{1}$ & $\mathrm{Cl}_{1}$ \\
\hline \multirow{2}{*}{ Mean $\pm \mathrm{SD}$} & $1.1049 \pm$ & $0.7568 \pm$ & $0.8193 \pm$ & $0.7203 \pm$ & $1.1122 \pm$ & $0.9809 \pm$ & $0.9551 \pm$ & $0.9809 \pm$ \\
& 0.1534 & 0.1253 & 0.1164 & 0.1023 & 0.2112 & 0.0985 & 0.1126 & 0.0985 \\
\hline
\end{tabular}

$\mathrm{N}_{1}, \mathrm{~S}_{1}, \mathrm{M}_{1}, \mathrm{I}_{1}$ : dimension with conventional technique at 1 day postoperative, $\mathrm{CN}_{1}, \mathrm{CS}_{1}, \mathrm{CM}_{1}, \mathrm{CI}_{1}$ : dimension with mini-implant and surgical wire at 1 day postoperative, SD: standard deviation.

Table 4. Fractal dimension selected at 1 day postoperative

\begin{tabular}{ccccccccc}
\hline & $\mathrm{N}_{2}$ & \multicolumn{1}{c}{$\mathrm{S}_{2}$} & $\mathrm{M}_{2}$ & $\mathrm{I}_{2}$ & $\mathrm{CN}_{2}$ & \multicolumn{1}{c}{$\mathrm{CS}_{2}$} & $\mathrm{CM}_{2}$ & $\mathrm{Cl}_{2}$ \\
\hline \multirow{2}{*}{ Mean $\pm \mathrm{SD}$} & $1.1118 \pm$ & $0.7947 \pm$ & $0.8697 \pm$ & $0.7362 \pm$ & $1.1771 \pm$ & $1.0123 \pm$ & $1.0003 \pm$ & $0.9905 \pm$ \\
& 0.1510 & 0.1197 & 0.1217 & 0.0986 & 0.2105 & 0.1253 & 0.1211 & 0.2093 \\
\hline
\end{tabular}

$\mathrm{N}_{2}, \mathrm{~S}_{2}, \mathrm{M}_{2}, \mathrm{I}_{2}$ : dimension with conventional technique after 5.8 weeks, $\mathrm{CN}_{2}, \mathrm{CS}_{2}, \mathrm{CM}_{2}, \mathrm{CI}_{2}$ : dimension with mini-implant and surgical wire after 5.8 weeks, SD: standard deviation. 
$0.7568 \pm 0.1253,0.8193 \pm 0.1164,0.7203 \pm 0.1023$, and $1.1049 \pm 0.1534$, respectively. Finally, the average fractal dimension values of $\mathrm{S}_{2}, \mathrm{M}_{2}, \mathrm{I}_{2}$, and $\mathrm{N}_{2}$ in the radiograph taken 5.8 weeks on the average after surgery were $0.7947 \pm 0.1197,0.8697 \pm 0.1217$, $0.7362 \pm 0.0986$, and $1.1771 \pm 0.2105$, respectively. The near areas $\left(\mathrm{CN}_{0}, \mathrm{CN}_{1}, \mathrm{CN}_{2}\right)$ of the right $\mathrm{M}$ on the fracture line--selected as ROI of the normal mandible of the patients to whom the mini-implant and surgical wire technique were applied--did not show any statistically significant difference according to time flow. $\mathrm{N}_{0}, \mathrm{~N}_{1}$, and $\mathrm{N}_{2}$ of the patients who underwent conventional open reduction and intra-osseous fixation did not show any statistically significant difference according to time flow.

All fractal dimension values of ROI on the fracture line (CI, CM, CL, I, M, L) showed significant differences $(\mathrm{P}<0.05)$ according to the time flow (first examination, 1 day after surgery, and average of 5.8 weeks after surgery), regardless of the surgery method (Tables 2 4).

\section{Changes in Fractal Dimension according to the Method}

In comparing $\mathrm{CS}_{0}$ and $\mathrm{S}_{0}, \mathrm{CM}_{0}$ and $\mathrm{M}_{0}$, and $\mathrm{CI}_{0}$ and $\mathrm{I}_{0}$ on the fracture line from the radiograph of the first examination, the average fracture dimension values were higher for the group of the patients who had mini-implant and surgical wire treatment. There was no statistically significant difference between the two groups, however. On the other hand, in comparing $\mathrm{CS}_{1}$ and $\mathrm{S}_{1}, \mathrm{CM}_{1}$ and $\mathrm{M}_{1}$, and $\mathrm{CI}_{1}$ and $\mathrm{I}_{1}$ on the fracture line from the radiograph 1 day after the surgery, the average fracture dimension values were higher for the group of patients who had miniimplant and surgical wire treatment; the difference between the two groups was statistically significant at 5\% significance level. Finally, in comparing $\mathrm{CS}_{2}$ and $\mathrm{S}_{2}, \mathrm{CM}_{2}$ and $\mathrm{M}_{2}$, and $\mathrm{CI}_{2}$ and $\mathrm{I}_{2}$ on the fracture line from the radiograph 5.8 weeks on the average after surgery, the average fracture dimension values were higher for the group of patients who had mini-
Table 5. Comparison of fractal dimension at each area (preoperative)

\begin{tabular}{cccc}
\hline & \multicolumn{3}{c}{ Fractal dimension at fracture line } \\
\cline { 2 - 4 } & Superior area & Middle area & Inferior area \\
\hline Group A & $0.7163 \pm 0.1407$ & $0.7120 \pm 0.1486$ & $0.6916 \pm 0.1230$ \\
Group B & $0.6966 \pm 0.1378$ & $0.6701 \pm 0.1501$ & $0.6737 \pm 0.1136$ \\
P & $>0.05$ & $>0.05$ & $>0.05$ \\
\hline
\end{tabular}

Group A: reduction with mini-implant and wire, Group B: conventional reduction.

Table 6. Comparison of fractal dimension at each area (postoperative)

\begin{tabular}{cccc}
\hline & \multicolumn{3}{c}{ Fractal dimension at fracture line } \\
\cline { 2 - 4 } & Superior area & Middle area & Inferior area \\
\hline Group A & $0.9675 \pm 0.1034$ & $0.9551 \pm 0.1126$ & $0.9809 \pm 0.0985$ \\
Group B & $0.7568 \pm 0.1253$ & $0.8193 \pm 0.1164$ & $0.7203 \pm 0.1023$ \\
P & $<0.05$ & $<0.05$ & $<0.05$ \\
\hline
\end{tabular}

Group A: reduction with mini-implant and wire, Group B: conventional reduction.

Table 7. Comparison of fractal dimension at each area (5.8 weeks)

\begin{tabular}{cccc}
\hline & \multicolumn{3}{c}{ Fractal dimension at fracture line } \\
\cline { 2 - 4 } & Superior area & Middle area & Inferior area \\
\hline Group A & $1.0123 \pm 0.1253$ & $1.0003 \pm 0.1211$ & $0.9905 \pm 0.2093$ \\
Group B & $0.7947 \pm 0.1197$ & $0.8697 \pm 0.1217$ & $0.7362 \pm 0.0986$ \\
P & $<0.05$ & $<0.05$ & $<0.05$ \\
\hline
\end{tabular}

Group A: reduction with mini-implant and wire, Group B: conventional reduction.

implant and surgical wire treatment; the difference between the two groups was statistically significant at $5 \%$ significance level (Tables 5 7).

\section{Discussion}

Methods for the internal fixation of mandibular fracture generally include wire ligation fixation, fixation using compression plate or reconstructive plate, and fixation using lag screw and miniplate. Nowadays, internal fixation using miniplate is widely used because of its simple usage as intra-oral surgery and excellent clinical results ${ }^{13)}$. Regardless of various fixation methods, however, 
the commonly important thing in all cases is to minimize the movement of fracture fragments with proper fixation to prevent complications such as infection or malunion after surgery. In other words, to prevent complications after surgery, the gap between fracture fragments should be minimized, and the contact area between fracture fragments should be maximized; thus, the fracture healing process that started from the primary callus (soft callus) reaction should proceed stably $^{14)}$. Note, however, that the displacement of fracture fragments in the mandibular fracture can be influenced by the direction of fracture line or the working direction of muscle on the fracture fragments as well as the direction and power of shock. In case of simple fracture, the proximal segment that includes the condyle receives tension in the gap direction by masseter muscle and medial/lateral pterygoid, and the distal segment receives tension by the genioglossus muscle. In addition, two fracture fragments tend to be separated by occlusal force imposed in various locations during mastication.

When using mini-implant and surgical steel wire, the temporary use of mini-implant and surgical steel wire before internal fixation can minimize the gap between fracture fragments. In the orthodontics field, the mini-implant has recently been drawing attention as a method of obtaining anchorage in the mandible since it minimizes the anchor loss of the molar. Since this has a dual-head manufactured for the orthodontic elastic to be hung, it is easy to hang surgical wire. Likewise because this reduces proximal and distal fracture fragments without gap for a short time, one can perform internal fixation when the gap between fracture fragments has almost disappeared.

In the healing of mandibular fracture, an important procedure for prognosis evaluation is observation through regular radiograph shooting after surgery. Panoramic radiograph is useful as an instrument for evaluating the gap between fracture fragments.
Although computed tomography can give threedimensional information, it is expensive; the high amount of radiation dosage also makes it difficult to obtain the patient's cooperation. Therefore, prognosis evaluation is usually made through panoramic radiograph. Chin et al. (radiographical evaluation of fracture line stability during functional loading after mini-plate fixation of mandibular angle fractures) have fixed fracture only with one mini-plate near the external oblique ridge--an ideal bone formation line suggested by Champy et al. ${ }^{5)}$--and immediately permitted its function without the period of intermaxillary fixation and subsequently measured and analyzed the fracture line stability and amount of disocclusion of inferior area in particular through radiographs that were shot regularly after surgery ${ }^{15)}$. Note, however, that Brynolf $^{16)}$, Goldman et al. ${ }^{17)}$, Bender and Seltzer ${ }^{18)}$ have reported the difficulty and discord of this macroscopic radiograph interpretation. This is because that the interpreter's interpretation standard depends greatly on subjective factors.

As a method for the quantification and objectification of interpretation to overcome the limit of macroscopic interpretation of radiograph, fractal analysis was introduced by Mandelbrot ${ }^{19)}$. Fractal analysis quantifies the structure showing self-similarity and evaluates values as a decimal level. Since it is a means of evaluating the complexity of structure, the fractal dimension gets bigger with increasing complexity. Changes in bone density and trabecular direction are nonlinear and disorderly. Nonetheless, fractal geometry expresses the characteristic of structures by quantifying them in geometric terms, although the fractures are neither equal nor regular".

Similarly, since the trabecular has fractal structure, the dentistry field has continuously tried to use fractal analysis in diagnosing osteoporosis and periodontal diseases. Lee et al. ${ }^{10)}$ have reported that fractal dimension decreased as the specimen of bovine lone bones gets decalcified. According 
to Khosrovi et al. ${ }^{11)}$, the fractal dimension of the alveolar bone of periodontal disease was less than that of normal alveolar bone. In addition, Southard et al. ${ }^{20)}$ reported that the fractal dimension after the decalcification of alveolar bone fragments was less than that before the decalcification. Cha et al. ${ }^{21)}$ showed that fractal analysis was useful in diagnosing periodontal diseases by identifying the difference between a normal person and a patient of periodontal disease in terms of the fractal dimensions in the alveolar bone of the downward disocclusion area of tooth root and alveolar crest and fractal dimension rate in the $1 / 3$ middle area of interdental bone. Lin et al. ${ }^{22)}$ quantified the structure form of spongy bone in the specimen of thigh bone and reported that fractal dimension had statistically significant correlation with bone intensity. Shrout et al. $^{23)}$ reported that the fractal dimension measured in digitalized dental radiograph was not influenced by the exposure time and diversity of radiation angle, and that the absolute location of ROI was not necessary. Another study reported that fractal dimension could be calculated in a non-standardized clinical radiograph, and that it could be used in distinguishing gingivitis and periodontitis $^{24)}$. In this study, too, we could see that fractal figures of ROI on the fracture line in the radiographs after mandibular open reduction and intra-osseous fixation had increased, and the difference was statistically significant.

Moreover, research studies on the factors that can influence the result of fractal analysis are rigorously conducted. Shrout et al. ${ }^{23)}$ reported that the size and form of ROI could influence the result of fractal analysis, contrary to previous studies. Likewise, Chen and Chen ${ }^{25)}$ said that fractal dimension showed statistically significant changes depending on the shooting angle. According to Veenland et al. ${ }^{26)}$, the fractal dimensions measured in the radiograph could be compared only when the same subjectfocal distance, same exposure condition, and same digital device of the same resolution were used.
Pornprasertsuk et al. ${ }^{27)}$ showed the difference depending on the recording media by reporting that fractal dimension values measured in digital film were higher than those measured in CCD. Southard et al. ${ }^{20)}$ explained that the difference in the calculation method of fractal dimension values and the difference in experimental subjects could yield contradictory results. Still, what is certain is that fractal analysis can be a quantitative, objective method as an indicator of bone changes.

The results above showed that fractal dimension values on the fracture line to which mini-implant and surgical steel wire were applied had increased compared with conventional open reduction and intra-osseous fixation, and the difference was statistically significant. Based on this, the reduction technique using min-implant and surgical steel wire can be regarded as an effective method of minimizing the gap between mandibular fracture fragments.

\section{Conclusion}

Based on the results of the study on the usefulness of the reduction technique using mini-implant and surgical steel wire in the treatment of mandibular fracture through the fractal analysis method, the reduction technique using min-implant and surgical steel wire is regarded as an effective method of minimizing the gap between mandibular fracture fragments.

\section{References}

1. Spiessl B, Rahn B: Internal fixation of the mandible: a manual of AO/ASIF principles. Berlin, New York: Springer-Verlag; 1989.

2. Park YA, Sohn WI, Chang IJ, Song JC, Chin BR. Complications associated with open reduction of mandibular fractures. J Korean Assoc Oral Maxillofac Surg. 2001; 27: 474-80.

3. James RB, Fredrickson C, Kent JN. Prospective study of mandibular fractures. J Oral Surg. 1981; 
39: $275-81$.

4. Sawaizumi M, Maruyama Y, Onishi K. Use of temporary miniplate for fixation in cases of mandibular fracture. J Craniofac Surg. 1995; 6: 417-20.

5. Champy M, Loddé JP, Schmitt R, Jaeger JH, Muster D. Mandibular osteosynthesis by miniature screwed plates via a buccal approach. J Maxillofac Surg. 1978; 6: 14-21.

6. Yang BE, Choi YJ, Choi WC. Effective reduction of mandibular angle fracture with Mini-implant; case report. J Korean Assoc Oral Maxillofac Surg. 2007; 33: 397-400.

7. Yaşar F, Akgünlü F. The differences in panoramic mandibular indices and fractal dimension between patients with and without spinal osteoporosis. Dentomaxillofac Radiol. 2006; 35: 1-9.

8. Yasar F, Akgünlü F. Fractal dimension and lacunarity analysis of dental radiographs. Dentomaxillofac Radiol. 2005; 34: 261-7.

9. Weibel ER. Fractal geometry: a design principle for living organisms. Am J Physiol. 1991; 261: L361-9.

10. Lee KI, Choi SC, Park TW, You DS. Fractal dimension calculated from two types of region of interest. Dentomaxillofac Radiol. 1999; 28: 284-9.

11. Khosrovi PM, Kahn AJ, Majumdar HK, Genant CA. Fractal analysis of dental radiograph to assess trabecular bone structure. J Dent Res. 1994; 73: 173.

12. White SC, Rudolph DJ. Alterations of the trabecular pattern of the jaws in patients with osteoporosis. Oral Surg Oral Med Oral Pathol Oral Radiol Endod. 1999; 88: 628-35.

13. Michelet FX, Deymes J, Dessus B. Osteosynthesis with miniaturized screwed plates in maxillo-facial surgery. J Maxillofac Surg. 1973; 1: 79-84.

14. Nakamura S, Takenoshita Y, Oka M. Complications of miniplate osteosynthesis for mandibular fractures. J Oral Maxillofac Surg. 1994; 52: 233-8.

15. Suh CH, Bae JS, Chin BR. Radiological evaluation of fracture line stability during functional loading after miniplate fixation of mandibular angle fractures. J Korean Assoc Oral Maxillofac Surg. 2001; 27: 428-34.
16. Brynolf I. Radiography of the periapical region as a diagnostic aid. I. Diagnosis of marginal changes. Dent Radiogr Photogr. 1978; 51: 21-39.

17. Goldman M, Pearson AH, Darzenta N. Endodontic success--who's reading the radiograph? Oral Surg Oral Med Oral Pathol. 1972; 33: 432-7.

18. Bender IB, Seltzer S. Roentgenographic and direct observation of experimental lesions in bone: part I. J Am Dent Assoc. 1961; 62: 152-60.

19. Mandelbrot BB: The fractal geometry of nature. New York: Freeman cop; 1983.

20. Southard TE, Southard KA, Jakobsen JR, Hillis SL, Najim CA. Fractal dimension in radiographic analysis of alveolar process bone. Oral Surg Oral Med Oral Pathol Oral Radiol Endod. 1996; 82: 569-76.

21. Cha SY, Han WJ, Kim EK. Usefulness of fractal analysis for the diagnosis of periodontitis. Korean J Oral Maxillofac Radiol. 2001; 31: 35-42.

22. Lin JC, Grampp S, Link T, Kothari M, Newitt DC, Felsenberg D, Majumdar S. Fractal analysis of proximal femur radiographs: correlation with biomechanical properties and bone mineral density. Osteoporos Int. 1999; 9: 516-24.

23. Shrout MK, Potter BJ, Hildebolt CF. The effect of image variations on fractal dimension calculations. Oral Surg Oral Med Oral Pathol Oral Radiol Endod. 1997; 84: 96-100.

24. Shrout MK, Roberson B, Potter BJ, Mailhot JM, Hildebolt CF. A comparison of 2 patient populations using fractal analysis. J Periodontol. 1998; 69: 9-13.

25. Chen SK, Chen CM. The effects of projection geometry and trabecular texture on estimated fractal dimensions in two alveolar bone models. Dentomaxillofac Radiol. 1998; 27: 270-4.

26. Veenland JF, Grashius JL, van der Meer F, Beckers $\mathrm{AL}$, Gelsema ES. Estimation of fractal dimension in radiographs. Med Phys. 1996; 23: 585-94.

27. Pornprasertsuk S, Ludlow JB, Webber RL, Tyndall DA, Yamauchi M. Analysis of fractal dimensions of rat bones from film and digital images. Dentomaxillofac Radiol. 2001; 30: 179-83. 